\title{
Some Problems in the Corpus Selection of Chinese Grammar in Spring and Autumn Period
}

\author{
Weiming Peng \\ College of Liberal Arts \\ South China Normal University \\ Guangzhou, China 510006
}

\author{
Jianya Zhang \\ College of Liberal Arts \\ South China Normal University \\ Guangzhou, China 510006
}

\begin{abstract}
Since King Ping of Zhou moved forward the eastern of China, it was known as the Spring and Autumn period. The written Chinese language handed down from generation to generation - the form and structure of the classical Chinese has been basically shaped during this period. And it belongs to the "model period of classical Chinese". In particular, it is of great significance to study Chinese grammar in this early period (the Spring and Autumn period). This paper discusses the problems in corpus selection process of the Chinese grammar research from four aspects. First, we should promptly replenish the newly released unearthed documents and take it as the corpus of the study. Secondly, it should attach importance to the corpus with many components of spoken language. Thirdly, hysteretic and solemn written language should not be ruled out. Although these languages lack the context of dialogue, they are also one of the actual features of the Chinese in Spring and Autumn period. Fourth, we must carefully select the corpus, and get rid of controversial literature handed down from generation to generation. The scholars should study the Chinese in Spring and Autumn period. And they should know what corpus can meet the needs of linguistic research. This would have a very important enlightenment on the study of the history of Chinese grammar.
\end{abstract}

Keywords-the Chinese in Spring and Autumn period; research on grammar; corpus selection

\section{INTRODUCTION}

First of all, we need to know what the Chinese is in Spring and Autumn period? Since King Ping of Zhou moved forward the eastern, it was called as the Spring and Autumn period by the Chinese historians. The so-called Spring and Autumn period originated from "The Spring and Autumn Annals" of the history of Lu. It started in the first year of Duke Yin of Lu (722 BC) and ended in the fourteenth year of Duke Ai (481 BC). Later, for the convenience of narration, the Spring and Autumn period was written 770 BC to 476 $\mathrm{BC}$ (And the first year of King Ping of Zhou was the beginning of the Eastern Zhou dynasty. And it ended in the 44th year of King Jing of Zhou) [1]. During this period, due to the frequent contacts among the vassal states and the deepening intercourse of ethnic cultures, it was also the period when great changes took place in the society. From the Bronze Age to the Iron Age, from the collapse of the slavery system to the establishment of the feudal system, the culture was prosperous. And the thinking was active. A hundred flowers bloom and a hundred schools of thought contend. Thus, regional common language of the Han nation is mature, which was called "Mandarin". As the official school began towards the public, private learning was flourishing. With broad dissemination and learning of the "Book of Songs", "Book of Rites" and "Etiquette", the internal laws of common language "Ya-yan" gradually stabilized. The written Chinese language handed down from generation to generation - the form and structure of the classical Chinese language had basically been shaped during this period. The linguists call this period "a model period for classical Chinese".

Second, what are the characteristics of the Chinese grammar in Spring and Autumn period? We could have a brief description. First, the judgment sentence composed by the judgement word "维" becomes the main form of judgment sentence. Also, it is the main usage of the word. There are 262 words of "维" in the "Book of Songs". And there are 98 words in the usage of judgments, accounting for one third. Second, as auxiliary words, "为" and "见" were put before the verb. It is the passive form, appearing in this period [2] ${ }^{385}$. "为" would draw forth something to do. Also, " 为 " is often placed before the verb. Third, question and modal words and associated words of Chinese are generated and developed [2] ${ }^{415}$. The above three points can't cover all the linguistic features of the Chinese in Spring and Autumn period. However, the principles of Chinese in the Spring and Autumn period can be explored. Its significance is enormous.

Third, what Chinese documents belong to the corpus of the Spring and Autumn period? The current academic response to this question is still not uniform.

In his article "research on the third personal pronouns of unearthed and handed-down documents in the Spring and Autumn period", Zhang Yujin made a macro definition and explanation of the corpus selection of the Chinese in Spring and Autumn period [3]. The materials of the Spring and Autumn period can be divided into two categories. One is the handed-down documents. And the other is unearthed literature.

There are three main handed-down documents. First, it is the "lyrics" in the "Book of Songs" (among which, it may be the literature in Western Zhou dynasty), "Ode to Shang", and "Ode to Lu". The vast majority of this corpus was in the 
early Spring and Autumn period and the middle of Spring and Autumn period. Second, it is "life of Hou" and "Qin oath" in "Shangshu". These two documents are also in the early Spring and Autumn period. Third, it is "The Spring and Autumn Annals", which is the original text of Lu history in the Spring and Autumn period.

Unearthed literature is mainly inscriptions on ancient bronze objects in Spring and Autumn period. This corpus mainly could be seen in the following two books. First, it is "The Collection of Inscriptions on Bronzes in Yin \& Zhou". Second, it is "The Collection of New Inscriptions on Bronzes in Yin \& Zhou". The unearthed literature includes the wooden tablets of "Book of Songs" in Han dynasty in Fuyang. It is incomplete. However, it still has reference value. When the scholars study the language of "Book of Songs", they should study it.

The author believes that Zhang Yujin's research on the Chinese corpus selection and classification in Spring and Autumn period is reasonable. He dose pay attention to the corpus value of the excavated literature and the handeddown documents. Also, he takes into account of the spoken language, the written language and the verse style. It has many sources of corpus. The type is not uniform. All types have the reasonable proportion. Of course, some scholars have raised objections to the views of Zhang Yujin. The special discussion is shown in the following.

\section{To Supplement NeWly UneARTHED DocumeNTS TIMELY}

The academic achievements in each era have been more innovative than the previous one. Of course, some new ideas and the results are also subject to the limitations of the academic circles in this era. The scholars should timely replenish the newly released unearthed literature. In summary, there are two main aspects.

First, the study of Chinese grammar in Spring and Autumn period must pay attention to the corpus which has been completely published by practitioners. Taking the "oath instrument" of the stone and jade inscriptions recording alliances from Houma of unearthed literature as an example, we know that in the Spring and Autumn period, the handeddown paper is very scarce. And the gold and stone inscriptions are especially valuable. A special style of alliance materials take jade tablets and stone tablets (some experts speculated that it used the remaining material of jade artifacts) as the carrier. Also, Zhu Shu and Mohist books are the main. And the content belongs to the oaths of Zhao Family of Jin dynasty in late Spring and Autumn period. And it is unearthed in Houma, Shanxi. And it is a site of Jin dynasty. So academics call this "Stone and Jade Inscriptions Recording Alliances from Houma". In the mainstream view of the academic circles, the editor of "Stone and Jade Inscriptions Recording Alliances from Houma" is Zhao Yang, the minister of Jin dynasty. In order to fight against the forces of the old aristocracy in Jin dynasties, Zhao Yang and the family made a joint oath to the world. They must keep the unity in the family. According to the record of the book, if the slaves got military exploit in the fight, they could get personal freedom and other material rewards. The discovery of "Stone and Jade Inscriptions Recording Alliances from Houma" is of tremendous significance. And it has great values for the research on archeology, history, ancient philology, and Chinese philology. It is also important corpus for studying the true appearance of Chinese language in the Spring and Autumn period.

Second, the study of Chinese grammar in Spring and Autumn period must also pay attention to the upcoming new corpus. According to the introduction of the president of the Chinese Literary Institute and professor Huang Dekuan of Anhui University, in early 2015, Anhui University recruited a group of bamboo slips written in the Warring States Period. The bamboo slips were made in the early and middle stages of the Warring States Period. And it is major discovery followed bamboo slips of Guodian, Chu Bamboo Slips Collected by Shanghai Museum, and the Chu Bamboo Slips of Tsinghua in the Warring States period. These bamboo slips are all ancient books and literature, involving the different subjects of Confucian classics, history, philosophy, literature and philology. And they include the slips of "Book of Songs". And they have deposited 97 slips. The content is "lyrics" of "Book of Songs". It contains 58 poems (including fragments). Among the simplified version of the "lyrics" and "Mao Poem", other handed-down texts and unearthed literature, there are a large number of variant characters [4]. Among these variant characters, the chapter order of "Book of Songs" is different. And the words and expressions are different. With the publishing of the slips of "The Book of Songs" in Anhui University, the ancient writing scholars constantly updated interpretation comments. More solid explanation of interpretation has been put forward. All these latest achievements provide extremely important conditions for studying the actual appearance of the Chinese grammar in Spring and Autumn period. Compared with the forthcoming slips of "The Book of Songs" in the Chu State, scholars who used to study the Chinese grammar in Spring and Autumn period can only reference the slips of "Book of Songs" in Fuyang, which has severe disability. The limitations of the study on the corpus of "lyrics" in "The Book of Songs" can be imagined.

However, due to the limitation of various factors such as the particularity of the unearthed texts and the early publication, there may have no breakthrough in the interpretation of the texts. With the announcement of the material, it may be limited to the current interpretation of the ancient text. There are still mistakes in the interpretation of the text. For the scholars, these are stumbling blocks to have study on the Chinese grammar.

Of course, Yang Qingzhong once said: "At present, the researches on the excavated documents are still in the early stages of writing, compiling, interpreting and annotating. Most of the descriptions of the textual meanings can only be described as a kind of hypothesis. It can be regarded as the academic works of an independent thought which have formed a school of its own. And we should listen all of these visions. [5] The above quotation requires that academics who have dynastic description of the language must pay close attention to the latest trends of the ancient writing academics, 
seek truth from facts, and make a dialectical analysis based on the latest research results.

\section{TO PAy AtTENTION TO THE CORPUS WITH ORAL MATERIALS IN HANDED-DOWN DOCUMENTS}

There is a distinction between spoken language and written language in any era. (As for the Yin and Shang dynasties, the author won't discuss whether there is only spoken language at that time.) Compared with written language, spoken language is freer. It is not restricted by some "rules" of written language. Therefore, spoken language is "non-standard" language relative to written language. Contemporary grammar theory tells us to emphasize the study of spoken language. The spoken language is the simplest and most common form of language. The study of language should understand the fact of language. In the process of recognizing the language activity, we should pay attention to the spoken language that people use in real life. Therefore, we must attach great importance to the corpus of the handed-down documents with much oral components. For example, the style of "The Book of Songs" may better reflect the actual spoken language at that time. The texts of "Life of Wenhou Hou" and "Qin Oath" in "Shangshu" belong to the normative written style, and pursue the elegant style of wording. Most of the articles focus on rhetoric rather than chronicle. Therefore, to a certain extent, it could reflect the actual spoken language.

In addition, when we use the corpus of unearthed documents, we also need to pay attention to the close relationship between colloquial level and genre type of the corpus. For example, in Qin bamboo slips, there is "Rishu" telling the good and bad fortune and Qin law documents. These corpus contents determine that the average usage of negative adverbs is obviously higher than that of other adverbs such as range adverbs and time adverbs. The reasons for the extremely unbalanced use of the sub-categories of these adverbs are probably related to their corpus style.

\section{HySTERESIS AND SOLEMN WRITTEN LANGUAGE SHOUld Not BE RulED OUT}

When we choose Chinese corpus of Spring and Autumn period, we should establish a principle of the rich and comprehensive types of corpus text. That is to say, we should not easily exclude hysteresis and solemn written language. When we choose the corpora, we need to consider some important contents. And we have to consider that which types of corpus could meet the needs of the language study.

In this section, the author would specially mention a scholar named Zhu Hong, who has different opinions from Zhang Yujin. In Zhu Hong's article "The study of firstperson pronouns in the Chinese of Spring and Autumn period", it took four handed-down documents such as "Etiquette", "lyrics", "national language" and "Zuozhuan" as the basic corpus for the study of Chinese in Spring and Autumn period [7]. However, in Zhu Hong's opinion, the handed-down documents of "Ode to Shang", "Ode to Lu", "Life of Wen Hou", "Qin oaths", "Spring and Autumn annual" and the inscriptions on ancient bronze objects of Spring and Autumn period do not fully reflect the actual appearance of the Chinese in Spring and Autumn period. These articles only can be as the reference for the study.

Zhu Hong's opinion has its merits. However, it is logically impracticable.

First of all, "The Spring and Autumn Annals" was edited by Confucius. The text is concise. And it lacks the dialogue context. And Zhu Hong excluded it. If "The Spring and Autumn Annals" is excluded from the scope of the Chinese corpus because of the conciseness of the text, the 15 articles of the "lyrics" in "The Book of Songs" belong to the verse, which is more concise than "The Spring and Autumn Annals". According to Zhu Hong's logical argument, it is unreasonable to take "lyrics" as the basic corpus.

Thirdly, Zhu Hong excluded the "Qin Oath" of "Shangshu". The literary text belongs to the "oath". The "oath" literature belongs to the words ancient kings told the warriors. The wording was not an improvisational speech. Therefore, there were differences with the actual Chinese at that time. The so-called "oaths" in "Shangshu" refer to the "oath words" (ie, the imperial edict language). And the emperor conducted the speech before the soldiers started the war. In the author's opinion, the "oath words" was to motivate the soldiers. At the time, due to the different ranks of soldiers' culture, the oath words should be simple and easy to understand. However, the scholars believe that the oath words are difficult to understand. It is impossible. "Oath word" comes from the ancient monarchs. They pursued elegant and concise language. And the words are easy to understand. It must be two important stylistic features.

The language of the imperial edict has been confirmed as a verbal language. In Lu Liehong's "corpus value of imperial edict", the corpus of the imperial edict showed that many imperial edicts actually used the new words and colloquialisms. And imperial edict has the same advantage as unearthed literature. It is extremely unlikely to be altered in the process of the spread. It belongs to the "simultaneous data" [8]. Therefore, in Zhu Hong's opinion, "Life of Wenhou" and "Qin Oath" used solemn written language. And this opinion is wrong.

In the end, Zhu Hong pointed out that the inscriptions on ancient bronze objects in the Spring and Autumn period belonged to the category of bronze inscriptions. The style was formal, and the language features were lagging. The authors believe that we can't arbitrarily identify the inscriptions on ancient bronze objects in the Spring and Autumn period of the unearthed literature as lagging languages. Due to the limitation of space, style and other factors, the inscriptions on ancient bronze objects in the Spring and Autumn period really can't fully reflect the language style in Spring and Autumn period. However, after all, the inscriptions on ancient bronze objects in the Spring and Autumn period belongs to the synchronic linguistic materials. Whether studying historical linguistics or the delineation of language, scholars have been conscious and able to use the corpus of the inscriptions on ancient bronze objects in the Spring and Autumn period better. This shows 
that the inscriptions on ancient bronze objects in the Spring and Autumn period and the so-called "oath" in "Shangshu" are similar in nature. It is written language. It is colloquial written language. It not only has the characteristics of written language, but also has colloquial style. It systematically reflects the actual appearance of the language in Spring and Autumn period. Therefore, in the process of studying the Chinese grammar in Spring and Autumn period, the corpus selection must avoid the depreciation of the corpus value of the inscriptions on ancient bronze objects in the Spring and Autumn period.

\section{TO EXClUdE CONTROVERSIAL HANDED-DOWN LITERATURE}

When we have the study on the Chinese in Spring and Autumn period, we need to attach importance to the handeddown documents and the unearthed documents. Some handed-down documents have serious disputes about the ownership of some times, such as "Rites", "Zhouli", "Laozi" and "Sun Tzu". Some people think that they are in the Spring and Autumn period. However, some people don't agree with this opinion. For example, some people think that the brewing of "Sun Tzu" is formed in Wu nation in the late Spring and Autumn period. However, the book is formed in the Warring States period. Also, some people think that "Etiquette" is formed in the Spring and Autumn period. And some people think that the book is formed in the late Warring States period [3]. For the controversial handeddown documents in this age, we would exclude them from the scope of the research corpus. And then, we could ensure that we would get true and reliable conclusions.

\section{CONCLUSION}

To sum up, the Spring and Autumn period is full of changes and struggles in Chinese history. During this period, the Chinese gradually became the model of the classical Chinese advocated by the ancient literati of later generations. When the scholars study the law of Chinese language in the Spring and Autumn period, they should pay attention to the choice of corpus first. The scholars should consider that which corpus could be the corpus of the Spring and Autumn period, and which document can't meet the needs. And then, the scholars should exclude them. The author noticed the above four aspects. First, the scholars should promptly replenish the new corpus. Secondly, they should attach importance to the corpus with spoken language. Third, the scholars shouldn't rule out the corpus of written language. Fourth, they should eliminate controversial corpus.

\section{ACKNOWLEDGEMENT}

This article is one of research results of the 2013 National Social Science Fund Project "Literature Research and Dictionary Compilation of Unearthed Documents in the Warring States Period" (No.:13BYY106). In the writing process, the author has got the help of Professor Zhang Yujin of college of arts of South China Normal University. Also, associate professor Zhang Yanmei of Guilin University of Electronic Science and Technology makes some suggestions.

\section{REFERENCES}

[1] Gao Xiaofang, Jiang Laidi. The corpus linguistics of Chinese history [M]. Beijing: Higher Education Press, 2005: 74.

[2] Wang Li. Wang Li collection (vol. 11). The history of Chinese grammar [M]. Jinan: Shandong Education Press, 1990.

[3] Zhang Yujin. Study on the third person pronouns of unearthed and handed-down documents in the Spring and Autumn period [J]. Chinese Writing Research, 2011: 84.

[4] Huang Dekuan. An overview of bamboo slips in the Warring States period of Anhui university [J]. Heritage, 2017 (9): 54.

[5] Yang Qingzhong. Unearthed documents and the reconstruction of ancient history [M]. Guilin: Lijiang Publishing House, 2012: 342.

[6] LI Mingxiao, Hu Bo, Zhang Guoyan. Study on the simple words in the Warring States and Qin and Han dynasties [M]. Chengdu: Sichuan University Press, 2011: 252.

[7] Zhu Hong. Study on the first person pronouns of the Chinese in Spring and Autumn period [J]. Chinese Research, 2012.

[8] Lu Liehong. The corpus value of imperial edict [J]. Journal of Chinese Social Science · 3rd Edition: Linguistics, 2017.10.24. 